\title{
Tidal disruption of stripped red giants by massive black holes
}

\author{
Y. Lu ${ }^{1}$, Y. F. Huang ${ }^{2}$, S. N. Zhang ${ }^{3}$ and P. Lu ${ }^{1}$ \\ ${ }^{1}$ National Astronomical Observatories, Chinese Academy of Sciences, Beijing 100012, China \\ email: ly@bao.ac.cn \\ ${ }^{2}$ Department of Astronomy, Nanjing University, Nanjing 210093, China \\ ${ }^{3}$ Physics Department and Center for Astrophysics, Tsinghua University, Beijing 100084, China
}

\begin{abstract}
We investigate the tidal disruption of a red giant whose envelope is thought to be stripped off when it passed by a massive black hole. Since the low-density stellar envelope would be lost, the tidal disruption of a red giant by massive black hole is regarded as primarily happening in its core region. The object is called a stripped red giant (SRG). Comparing our results with the three candidate tidal disruption events detected by Chandra in 2001 and 2002, i.e., the X-ray flares of NGC 5905, RX J1242.6-1119A, and RX J1624.9+7554, we argue that the tidal disruption of a stripped red giant is strongly ruled out.
\end{abstract}

Keywords. Accretion: accretion disks, black hole physics, galaxies: nuclei

\section{Introduction}

One remarkable sign of black holes residing in the centers of galaxies is the tidal disruption of stars by the black hole (Rees 1998). This provides a uniquely accessible laboratory for studying in detail the connections and interactions between a massive black hole and the stellar system (Alexander 2005, Lu et al. 2003, Lu et al. 2006). The ROSAT All-Sky Survey in 1990-1991 performed an experiment to detect these tidal disruption events with hundreds of thousands of galaxies in the ideal wavelength band. The results show that three galaxies, i.e., RX J1242.6-1119A, RX J1624.9+7554, and NGC 5905, had unusual X-ray flares. Interestingly, later observations of these galaxies by Chandra in 2001 and 2002 showed that their X-ray fluxes continued to decline at a rate consistent with the prediction in the fallback phase of a tidal disruption event (Gezari et al. 2003).

It has been discussed that the tidal disruption of a red giant and a main sequence star could be very different due to their different properties of the stellar structures (Lidskii \& Ozernoi(1979)). In this paper, we concentrate on the stripped red giant (SRG) in which only the core of a red giant, which is about 20 percent of the total mass (Luminet \& Barbuy(1990)) happens the tidal disruption.

\section{Tidal disruption of a stripped red giant}

When a star with a given mass of $M_{*}$ and radius of $R_{*}$ passes by a massive black hole with a mass of $M_{b h}$, the star would be tidally disrupted at an average tidal radius of $R_{t}=7 \times 10^{12} r_{*} m_{*}^{-1 / 3} M_{6}^{1 / 3} \mathrm{~cm}$, where $m_{*}=M_{*} / M_{\odot}, r_{*}=R_{*} / R_{\odot}$, and $M_{6}=$ $M_{b h} / 10^{6} M_{\odot} . R_{\odot}$ and $M_{\odot}$ are the solar radius and mass, respectively. Once a SRG is tidally disrupted by the black hole, the resulting luminosity of the X-ray flares can be approximated as $L_{X}=\epsilon \dot{M}_{\text {peak }} c^{2}$, where $\epsilon=5.38 \times 10^{-3} r_{*}^{-1} m_{*}^{1 / 3} M_{6}^{2 / 3}$ and $\dot{M}_{\text {peak }} \simeq$ $1.4 r_{*}^{-3 / 2} m_{*}^{2} M_{6}^{-1 / 2} M_{\odot} y r^{-1} . L_{x}$ shows that it depends on $\dot{M}$ and $\epsilon$. Given the mass of black holes, $L_{x}$ is determined by the accretion configuration and the property of disrupted 
stars. Throughout this discussion we use the term red giants loosely to describe progenitor stars on the first red giant branch, and we adopt the red giant model of (Han et al. (2000)) for the evolution of progenitor stars with masses in the range of $1 M_{\odot}<M_{G}<3 M_{\odot}$. We define the radius of SRGs by corresponding to the mesh point making the hydrogen-shell burning boundary and its mass by the onset of helium burning, respectively. we derive the mass-radius relation of SRGs as

$$
m_{S}=\left\{\begin{aligned}
53.11 r_{S}^{2}-3.29 r_{S}+0.37, & \text { if } 0.035 \leqslant \mathrm{r}_{\mathrm{S}} \leqslant 0.07756 \\
-16.77 r_{S}+0.95, & \text { if } 0.02819 \leqslant \mathrm{r}_{\mathrm{S}} \leqslant 0.035
\end{aligned}\right.
$$

where $m_{S}=M_{S R G} / M_{\odot}, r_{S}=R_{S R G} / R_{\odot}$. In the following calculations, we only consider those galaxies hosting massive black holes (i.e. $M_{b h} \geqslant 10^{6} M_{\odot}$ ) and those with massive black holes not massive enough to swallow a whole star without disruption. We thus ignore the tidal disruption of stars with the mass-radius relation as the lower branch of Eq.(2.1), because the corresponding upper limit of black hole mass is lower than $10^{6} M_{\odot}$, which is the typical lower mass limit for the black hole residing in the Galactic nucleus.

Chandra observations in the 0.1-2.4 keV band (Halpern et al.(2004)) show that the peak luminosity of the three flare events in NGC 5905, RX J1242.6-1119A and RX J1624.9+7554 are $4 \times 10^{43} \mathrm{erg} / \mathrm{s}, 1.6 \times 10^{44} \mathrm{erg} / \mathrm{s}$ and $4 \times 10^{43} \mathrm{erg} / \mathrm{s}$. With the prediction of the recent multi-wavelength observations that the black holes residing in these three tidal events are $\sim 1.7 \times 10^{8} M_{\odot}($ Gezari et al. $(2003)), \sim 2 \times 10^{8} M_{\odot}($ Ferrarese \& $\operatorname{Merritt}(2000))$ and $\sim 10^{6} M_{\odot}$ (Grupe et al.(1999)), we find that the luminosity calculated from the tidal disruption of a SRG star is too faint to account for the observations.

\section{Conclusions}

we have investigate the tidal disruption of SRGs by massive black holes. If this is the case, the results show it is ruled out for the three disruption events by comparing with the observations detected by Chandra in the 0.1-2.4 keV band.

\section{Acknowledgements}

This research is supported by the National Natural Science Foundation of China (Grants 10273011, 10573021, 10433010, 10625313, 10521001 and 10221001), and by Chinese Academy of Science through project No. KJCX2-YW-T03.

\section{References}

Alexander, T. 2005, Physics Reports 419, 65

Ferrarese, L. \& Merritt, D. 2000, ApJ 539, L9

Frank, J. \& Rees, M. 1976, MNRAS 176, 633

Gezari, S., Halpern, J. P., Komossa, S., Grupe, D., \& Leighly, K. M. 2003, ApJ 592, 42 (erratum $601,1159,[2004])$

Grupe, D., Thomas, H. C., \& Leighly, K. M. 1999, A\&A 350, L31

Halpern, J. P., Gezari, S., \& Komossa, S. 2004, ApJ 604, 572

Han, Z., Tout, C. A., \& Eggleton, P. P. 2000, MNRAS 319, 215

Lidskii, V. V. \& Ozernoi, L.M. 1979, Sov. Astron. Lett. 5, 16

Loeb, A. \& Ulmer, A. 1997, ApJ 489, 573

Lu, Y., Cheng, K. S., \& Zhang, S. N. 2003, ApJ 590, 52

Lu, Y., Cheng, K. S., \& Huang, Y.F. 2006, ApJ 641, 288

Luminet, J. P. \& Barbuy, B. 1990, AJ 61, 219

Rees, M. J. 1988, Nature 333, 523 